\title{
THE USE OF CAUSAL MAPS AS INTERDISCIPLINARY DIDACTIC REDUCTION METHOD
}

\author{
Daniyil Shmatkov \\ Ukrainian Engineering Pedagogics Academy, Kharkiv, Ukraine \\ d.shmatkov@uipa.edu.ua
}

\begin{abstract}
Presented article is devoted to theoretical substantiation and development of interdisciplinary didactic reduction method, based on the use of causal maps. The article discusses properties of causal maps in the context of its use in teaching methods. It was determined that the creation of the causal maps that reflect all connections between and / or within the three information blocks should be an effective reduction method of learning content of the topics about measurements of the various sciences. The blocks are named: "The physical basis of measurement", "The elements of the measuring instrument" and "The measurement technique". The article establishes that the method of using of causal maps in related topics of natural sciences, engineering, social sciences and humanities corresponds to properties of didactic reduction methods, due to abstraction and providing the omission of factors that do not have a determining influence on the situation, and getting in the vertices concepts less volume than learning texts. The method can provide an illustrative or symbolic representation of complex information on causal maps, review situations based on known models. The research justifies the possibility of constructing of quantitative variables in formulas to their qualitative explanations and presentation of the relationship between them in the causal maps. The process of tasks' decision, that has different difficulty levels, using causal maps allows to influence on additional perception channels, and through the students' understanding of causality allows intensifying the development of mental processes.
\end{abstract}

Keywords: didactic reduction; causal maps; interdisciplinary method; casual learning; teaching; measurement.

\section{Introduction}

The dynamic development of science, engineering and technology, and a high rate of information society dictate a significant increase in actual theoretical and empirical data from all courses in both secondary and higher education. Regulatory requirements for teaching time and a different level of students' intellectual abilities need to be supported by teaching methods that ensure coordination between the efficiency of teaching and objective limitations that exist in the educational process.

In this context, we consider the adaptation of the philosophical ideas of reductionism to the educational process which is called "didactic reduction". This direction has been developing for a long time and is intended to provide the content, scientifically based on the transformation of the amount of learning, as opposed to random changes. The term was first introduced in 1967 by the German professor Gustav Grüner (Grüner, 1967). It determined that the reduction in relation to the learning content can be of vertical or horizontal nature. Reducing the amount of information is the vertical nature of the reduction, i.e. its quantitative component. Reducing the level of teaching is a horizontal nature or quality reduction component.

Key aspects of didactic reduction implementation in the current development of the educational process are systematised by Martin Lerner (Lehrner, 2012). Also, the scientist identified the following functions of didactic reduction as selection, simplification, and concentration. In addition, the author notes that the method may involve a curricular reduction and highlighting of main aspects of the content. The final conclusion brings together different approaches to understanding didactic reduction.

Let's consider the current scientific developments in terms of didactic reduction of various courses contents. The group of scientists within this area has developed a software tool "to help the students in their approach to the study of graph algorithms, by guiding them to understand their logical structures through experiments on numerical instances" (Costa, D'Ambrosio \& Martello, 2014, p. 1115). The authors note that the software tool simplifies the perception of the subject area by its rational reducing.

Gerald Futschek proposes the use of "Extreme Didactic Reduction" (extreme degree of reduction) while studying data structures, programming or data modelling (Futschek, 2013). The scientist notes that for more educational results at the end of the process, it is necessary to reduce details of the learning content at the beginning. Futshek (2013) identified a function of didactic reduction, which is accelerating access to basic aspects of the topics.

To use modelling means as didactic reduction while writing textbooks on physics was offered by other scientists (Forjan \& Sliško, 2014). They offered the methods of reducing the complexity of learning such concepts as particle model, free fall, Hooke's law, the law of friction etc. The authors proved that a large amount of information in academic literature may be rejected without reducing the effectiveness of teaching. It focuses on the analysis of the most important physical phenomena by their idealisation. In addition, Forjan 
\& Sliško (2014) in reviewing the curriculum note that "...in the operative objectives the simplifications and idealisations are very poorly represented" (p. 28).

Other scientists during the analysis of teaching materials indicate that "some of the Bengali medium textbooks explained the conceptual knowledge nicely. But, in several cases, it was explained using (high level) mathematics, ignoring the conception of didactic reduction methodologies" (Haolader, Ali \& Foysol, 2015, p. 108).

Special attention in scientific works is paid to teaching the sciences of measurement. Ruhm (2011) offers empirical and analytical approaches that use appropriate mathematical models based on Signal and System Theory. According to the theory, mathematical models can contain both quantitative variables and qualitative. The Author thoroughly proves that reduction approach simplifies the analysis of the subject area and contributes to the general understanding of structural issues in the area. The study discusses the possibility of adapting the direction to any areas of science that use measurement.

Thus, the experience of experts indicates the great opportunities of approbation in the domestic pedagogical practice approach to learning the topics about measurements in various courses which contain a significant number of laws, formulas, relationships, patterns, techniques, etc. Application of didactic reduction method in the educational process provides an efficient distribution and isolation of learning material, reducing the overload of students by challenging theory, increasing the efficiency of understanding of fundamental phenomena, providing quick access to key aspects of the topics. All these benefits are provided to reduce the cognitive complexity of learning.

But most of these developments, despite the high efficiency, are described regarding specific subject areas. And multidisciplinary approach proposed by Ruhm (2011) requires teachers' knowledge about some aspects of Signal and System Theory, which complicates its implementation.

Presented article is devoted to theoretical substantiation and development of interdisciplinary didactic reduction method of the content of the topics about measurements in various courses based on the use of causal maps. This method should ensure the effectiveness of teaching and does not require special teachers' knowledge and skills.

\section{The theory of the using of causal maps in teaching}

Topics about different measurements are presented in all natural and engineering sciences and in such social and human sciences as anthropology, archeology, geography, criminology, art, education, psychology and so on. Therefore, methods aimed to increase the efficiency of teaching general topics about measuring in various courses might be called interdisciplinary.

As in the considered article (Ruhm, 2011), the proposed method is based on the principle of cause and effect relationships between processes. The relevance of applying the method based on causal maps has been highlighted by a large number of scientific works about various teaching forms of children and young people using causal relationships: "A theory of causal learning in children: causal maps and Bayes nets" (Gopnik, Glymour, Sobel, Schulz \& Kushnir, 2004); "Causal Learning: Psychology, Philosophy, and Computation" (Gopnik \& Schulz, 2007), "Predictive student model supported by fuzzy-causal knowledge and inference" (Peña-Ayalaa, Humberto Sossa-Azuelad \& Cervantes-Péreze, 2012), "The methods of nondestructive testing teaching of the future engineers and pedagogues using causal networks" (Lazarev \& Shmatkov, 2014), "Causal Learning: Understanding the World" (Young, 2016) etc.

Causal maps are represented as graphs, curves which reflect the causal relationships between vertices. The process of filling causal maps by substantive content is based on knowledge extraction developments.

Let us consider its properties. The simplicity of map construction is the fact that to create a similar model it is enough for a developer to own any method of knowledge extraction and theoretical basis of its structure. Theoretical foundations of the structure of causal networks include a genetic human desire to establish causal relationships between events. Mapping does not require sophisticated technical means. The syntactic structure of causal maps based on graph theory is close to effective understanding it by the man and provides the syntactic adequacy. Availability of models is the ability to work with the model of all places to people of different intellectual level and physical capabilities.

In the context of subject areas that have been dynamically developed, the possibility of replenishment of knowledge model plays a critical role. In causal maps, the creating of new peaks as elements of any kind or new relationships in the current model does not require the full processing of a model, and is only of a local character. It provides its adaptability (the ability to replenish with new information) and durability (compliance with contemporary trends in the subject area over time).

The dynamic development of sciences provides its information capacity. The selection of knowledge, which means the content of the course, from explanations and interpretations provides opportunities of 
assimilation per unit of time a greater volume of information. The same property determines the allocation of knowledge, which has no effective training functions. Because of this causal maps can be called ergonomic and economic.

The ability to select the basic elements of knowledge and the definition of logical relationships between them mainly makes use of maps in teaching methodology. The apparatus of causal maps with semantic relations between information units allow to fully providing didactic using. Through comprehensive causeeffect relationship simplicity of construction, high adaptability and didactic using, available syntax representation, causal maps are universal, that is to say, they have ample opportunities of knowledge description from different subject areas.

The dimension of such models may be more effective than knowledge provided by experts or reference literature and textbooks. It also can be achieved by the selection of only subject knowledge. Based on the model with causal relationships, developing the whole system does not require particularly complex actions. All semantic models differ in such feature, and casual maps with the same type of causal relationships greatly simplify the task.

Interaction with other models and systems is one of the basic properties of causal maps. Thus, the method can freely enter or join another educational system and other educational systems can freely enter or join this method.

In the matter of knowledge connectivity, semantic maps have the greatest advantage because it is their main function. This is especially true to causal maps that contain the same type of relationships. Causal maps sufficiently reflect the semantics of the subject area, describe local and global goals, are formalised, systematic, invariant, natural and visual, provide sufficient rapidity of orientation. Let's consider the use of causal maps as didactic reduction method of the content of topics about measurements.

\section{The theory of the using of causal maps in teaching topics about measurements}

The content of topics relating to the measurement of natural, technical, social and human sciences are clearly divided into three conventional blocks of knowledge that have cross-cutting nature of penetration through all learning information. Briefly, they can be called as following:

1) The physical basis of measurement;

2) The elements of the measuring instrument;

3) The measurement technique.

Each of the methods of measurement is based on the physical phenomena. They cover oscillatory and wave processes; electromagnetic field and radiation; laws of linear optics; thermal radiation of bodies; the structure of atoms and molecules; emission and absorption of energy by atoms and molecules; molecular physics and thermodynamics; physics of liquids and so on. This block can contain both physical processes and physical quantities which are known by the students.

The presence of the block "The elements of the measuring instrument" is a logical reflection of the area. It requires a number of basic skills and knowledge in the areas of measuring of geometric and mechanical quantities, measuring of temperature and pressure, measuring of physical and chemical composition and properties of substances, etc.

"The measurement technique" is an integral final part of the content. From this block, it is possible to refer knowledge of preparation for measurements to educational base and techniques of the performance of measurements and so forth.

The creation of causal maps that reflect all connections between and / or within the three information blocks should be an effective reduction method of learning content of the topics about measurements of the various sciences. It should also be noted that the vertices within a given block need not be ordered by the hierarchy. This is because the main objective is to cover relationships between individual facts and not their internal hierarchy.

Arcs that connect the vertices in causal maps are often represented as arrows from "cause" to "effect". It can be possible to supplement the space between the vertices that arcs occupying illustrations through appropriate means in an amount sufficient to maintain the planned teaching results. This improvement in the rational execution contributes to greater visualisation and consequently better perception.

The process of filling causal maps by substantive content is based on the design and implementation of algorithms for knowledge extraction by textual methods that provide assistance to young people in understanding the information presented in educational texts.

The standard causal map reflects the relationships between certain vertices of mentioned information blocks (Figure 1). Arcs between vertices should reflect causal relationships. The Concept A may be cause or 
effect of Concept B, Concept C, Concept D, Concept E, Concept F; Concept B may be cause or effect of Concept A, Concept C, Concept D, Concept E, Concept F and so on (presented in any form).

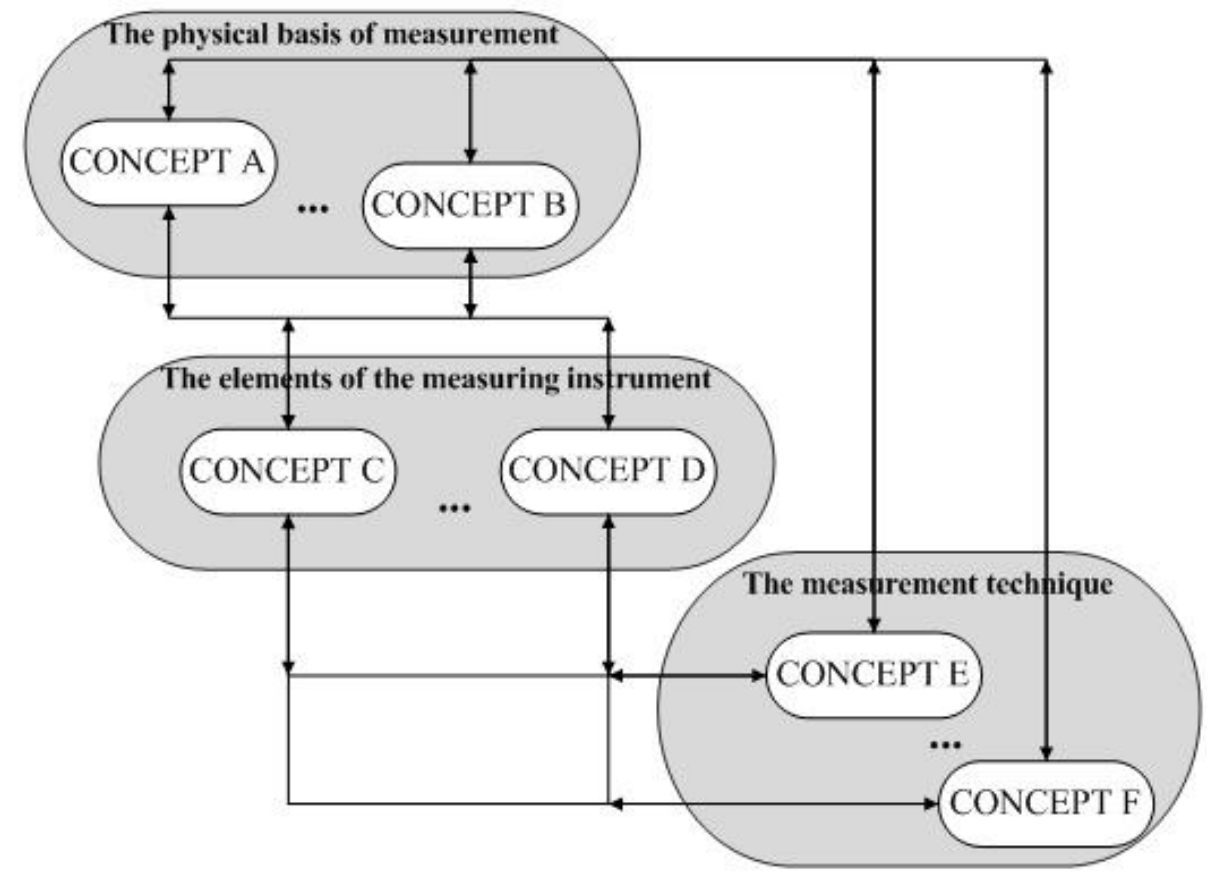

Figure 1. A theoretical model of the causal map.

A Graph with causal relationships implies that from any concept of any of the blocks there is the ability to move at least to two other concepts of two other blocks, or at least to two other concepts of one other block, or at least to one other concept of the same block. Movement (Figure 2) is possible as to the effects from the causes (following the arrow), and in the opposite direction. Causes and effects must obey certain situations. Such situations are modelled by the teacher. It appears such an opportunity in the formulation of questions and tasks of different difficulty levels (including the problem solving).

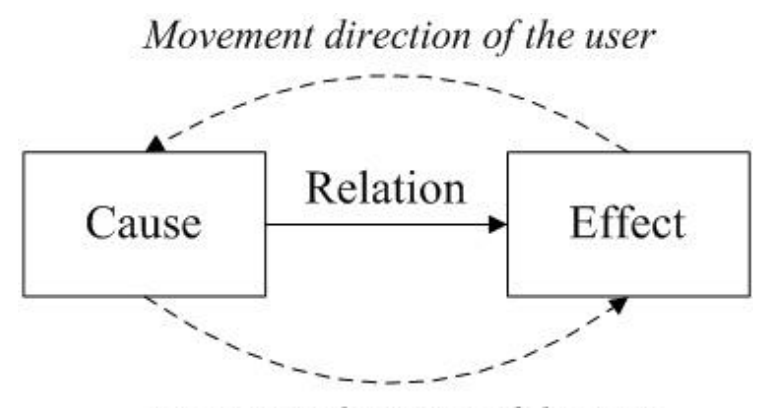

\section{Movement direction of the user}

Figure 2. Possible directions of movement through the causal map.

The practice of using causal maps in teaching topics about measurements

The implementation of this method requires the following actions from the teacher:

- identifying and working with reputable reliable sources;

- identifying the location of relationships of the triad: "The physical basis of measurement", "The elements of the measuring instrument" and "The measurement technique";

- determining causal relationships between keywords, representation of text structure in the shape of the graph;

- isolation with subsequent removal of concepts that are not in relationships with other concepts or do not reflect the specific of the topic;

- approbation of causal maps.

Let's consider a fragment of work with a causal map to the method of mass spectrometry. Such measuring device can be used in chemical, medical, criminology, environmental studies, etc. Let's construct 
a map that relates to leak test using a mass spectrometer, to the following thesis: "... as the test substances gases with low molecular mass are used..." (Figure 3).

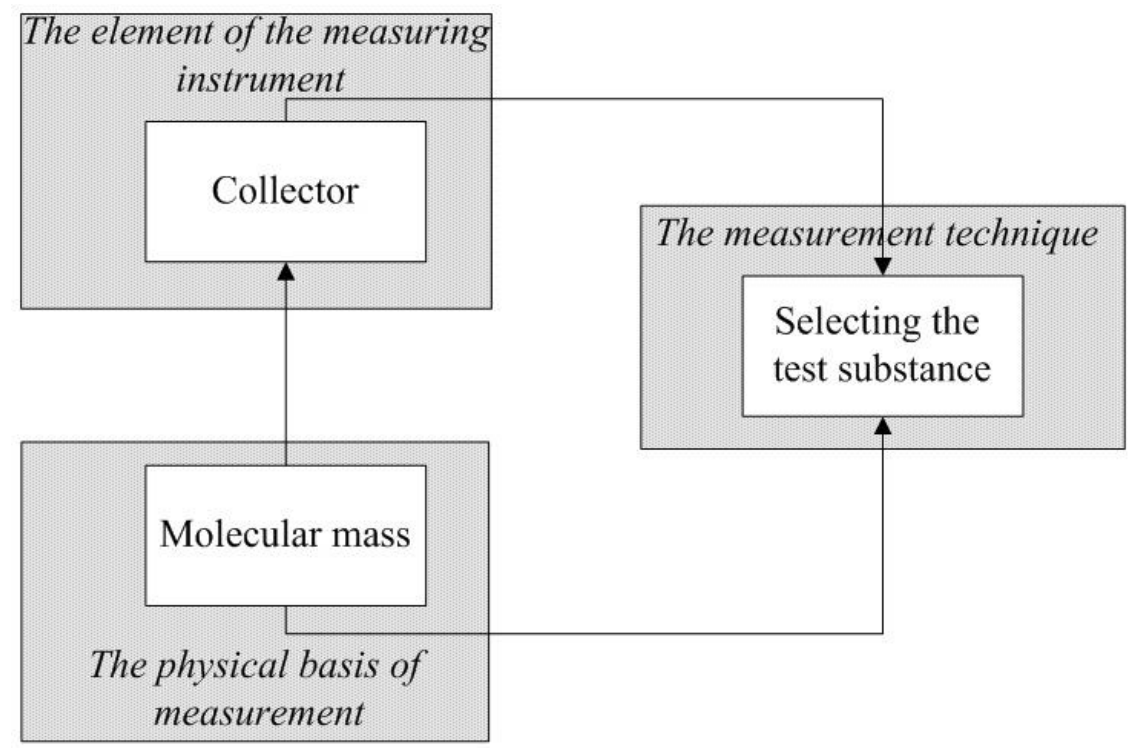

Figure 3. Fragment of causal map "Selecting test substance during a leak test".

Presented map shows only fragments of the subject area and is not exhaustive. There might be several maps to one issue. Factors affecting the particular vertices are determined by the teacher through the analysis of known information. As can be seen, the map visualises the concepts from three key information blocks and causal relationships between them are simple and understandable.

Now let's consider the solution of learning tasks using causal maps.

Let's analyse the following task: "Due to what can we screen out ions with different charges and mass to determine the amount of test substance at the output in conditions of mass spectrometric method?". The task is to acquaint students with the facts that have inexplicable character and have led to the formulation of the problem. To resolve this situation the information contained in the task must be clearly analysed. In the formulation of the question, the concepts that can be presented at the vertices of the other causal map are clearly identified as follows:"Ion mass" and "Ionic charge" from the block "The physical basis of measurement" and "Test substance" from the block "The measurement technique".

Then students are finding relationships with vertices of other blocks and determining their nature. The vertices "Ion mass", "Ionic charge" and "Test substance" must have a coincidence at the vertex "Collector", which belongs to the block "The elements of the measuring instrument". "Ion mass", "Ionic charge" and "Test substance" cause this vertex. So, students can come to the conclusion that the task of screening ions with different charges and mass needs to work on the configuration of the collector. It will be an attempt to find the right answer.

Let's analyse another task: "Is it possible for mass to realise leak test spectrometry without complying with the conditions of high vacuum?"

The algorithm, based on the analysis of other possible causal map determined that the vertex "Vacuum" should be connected with vertices "Ionizer" from the block "The elements of the measuring instrument" and "Mode of gas flow" from the block "The measurement technique". The vertex from the block "The physical basis of measurement" should cause the other two vertices. The key concept refers to one of three blocks. Therefore, considering the concept relationships with vertices of two other blocks it is possible to conclude that in this case, the vacuum is an important physical cause for measurement technique and for measuring instrument, so without complying with these conditions leak test is impossible. Thus students will be aware of the impossibility of taking some action on the basis of causal reasoning.

So, as mathematical models, causal maps can predict to a certain extent. The more relationships presented on a graph (especially bilateral), the more situations that cover maps are identified, and therefore their further development is easier to predict. Also using causal maps it is possible to represent complex formulas with relationships building based on the unknown quantity. 


\section{Conclusions}

Thus, the method of using causal maps that reflect the relationships between the physical basis of measurement, elements of the measuring instrument and features of the measurement techniques in the teaching of relevant topics of natural sciences, engineering, social sciences and humanities meets properties of the didactic reduction methods, as provides the following:

- abstraction and omission of factors that do not have a determining influence on the situation;

- getting in the vertices concepts less volume than learning texts;

- illustrative or symbolic representation of complex information on causal maps;

- review situations based on known models;

- construction of quantitative variables in formulas to their qualitative explanations and presentation of the relationship between them in the causal maps.

The method can provide both vertical and horizontal nature of the reduction, as well as a reduction in teaching hours and reducing the amount of information.

Construction of causal maps does not require special knowledge and skills from a teacher, but it requires a significant amount of time for analysing information sources and creating relevant graphs. The process of problem-solving, that has different difficulty levels, using causal maps allows influence over additional perception channels, and through the students' understanding of causality and allows intensifying the development of mental processes.

Prospects for further research could be the creation of algorithms, methods and means of optimising and automating the identification, construction and representation of causal relationships in educational texts of certain courses.

\section{References:}

Forjan, M., \& Slisko, J. (2014). Simplifications and Idealizations in High School Physics in Mechanics: A Study Of Slovenian Curriculum And Textbooks. European J of Physics Education, 5(3), 20-31. https://doi.org/10.20308/eipe.68867

Forjan, M., \& Slisko, J. (2014). Simplifications and idealizations in high school physics in thermodynamics, electricity and waves: A study of Slovenian textbooks. Latin-American Journal of Physics Education, 8(2), 241-247. Retrieved from http://lajpe.org/jun14/02_LAJPE_886\%20Forjan-Slisko.pdf

Futschek, G. (2013). Extreme Didactic Reduction in Computational Thinking Education. $X$ World Conference on Computers in Education. Retrieved from http://wcce2013.umk.pl/publications/Short_Papers/086-Futschek-SP-ext_msy.pdf

Costa, G., D’Ambrosio, C., \& Martello, S. (2014). Graphsj 3: A modern didactic application for graph algorithms. Journal of Computer Science 10 (7), 1115-1119. https://doi.org/10.3844/jcssp.2014.1115.1119

Gopnik, A. \& Schulz L. (Ed.). (2007). Causal Learning: Psychology, Philosophy, and Computation. New York, USA: Oxford University Press. https://doi.org/10.1093/acprof:oso/9780195176803.001.0001

Gopnik, A., Sobel, D. M., Glymour, C., Schulz, L. E., \& Kushnir, T. (2004). A theory of causal learning in children: causal maps and Bayes nets. Psychological Review, 111(1), 3-32. https://doi.org/10.1037/0033-295X.111.1.3

Grüner, G. (1967). Die didaktische Reduktion als Kernstück der Didaktik [The didactic reduction as the core of didactics]. Die Deutsche Schule 7/8, 414-430.

Haolader, F. A., Ali, M. R., \& Foysol, K. M. (2015). The Taxonomy for Learning, Teaching and Assessing: Current Practices at Polytechnics in Bangladesh and its Effects in Developing Students' Competences. International Journal for Research in Vocational Education and Training, 2(2), 99-118. https://doi.org/10.13152/IJRVET.2.2.2

Lazarev, M. \& Shmatkov, D. (2014). Metodyka navchannia neruinivnoho kontroliu maibutnikh inzheneriv-pedahohiv $z$ vykorystanniam kauzalnykh merezh [The methods of nondestructive testing teaching of the future engineers and pedagogues using causal networks]. Kharkiv, Ukraine: Tochka.

Lehner, M. (2012). Didaktische Reduktion [Didactic reduction]. Bern: Haupt.

Peña-Ayala, A., Sossa-Azuela, H., \& Cervantes-Pérez, F. (2012). Predictive student model supported by fuzzy-causal knowledge and inference. Expert Systems with Applications, 39(5), 4690-4709. Retrieved from https://doi.org/10.1016/j.eswa.2011.09.086

Ruhm, K. H. (2011). From Verbal Models to Mathematical Models - A Didactical Concept not just in Metrology. Joint International IMEKO TC1+ TC7+ TC13 Symposium. Retrieved from http://www.iwf.mavt.ethz.ch/ConfiguratorJM/publications/From Verba_132551321506614/IMEKOJenaRuhmII.pdf

Young, G. (2016). Causal Learning: Understanding the World. Unifying Causality and Psychology (pp. 387-415). Springer International Publishing. https://doi.org/10.1007/978-3-319-24094-7 16 